\title{
IMAGE OF THE MONTH
}

\section{Large pancreatic mass in a young woman}

\author{
E Rayner-Hartley $M D^{1}$, DF Schaeffer $M D^{2}$, F Donnellan MD ${ }^{1}$
}

A 26-year-old woman presented with several months' history of abdominal discomfort, postprandial bloating and nausea. The patient was otherwise well and had no significant medical or family history.

A computed tomography scan revealed a heterogeneous cystic and solid mass $14 \mathrm{~cm}$ in size in the right upper quadrant. There was no vascular involvement or lymphadenopathy, or biliary or pancreatic duct dilation (Figure 1A). Subsequent endoscopic ultrasound revealed a homogenous solid mass occupying most of the pancreas parenchyma (Figure 1B). Fine-needle aspiration revealed abundant tumour cells, characterized by granular cytoplasm and round to oval nuclei with finely textured chromatin and an indistinct nucleolus (Figure 1C); in areas, the tumour cells surrounded delicate hyalinized fibrovascular cores (Figures 1C and 1D). The tumour cells showed strong nuclear immunoreactivity for beta-catenin (Figure 1E).

\section{DISCUSSION}

Solid pseudopapillary neoplasms (SPNs) are the rarest of the four subtypes of pancreatic cystic neoplasms, representing $1 \%$ to $3 \%$ of all pancreatic tumours and $10 \%$ to $15 \%$ of all pancreatic cystic neoplasms. This neoplasm was first reported in 1959 , was subsequently classified by the WHO as a solid pseudopapillary tumour in 1996 and is now classified as SPN (1).
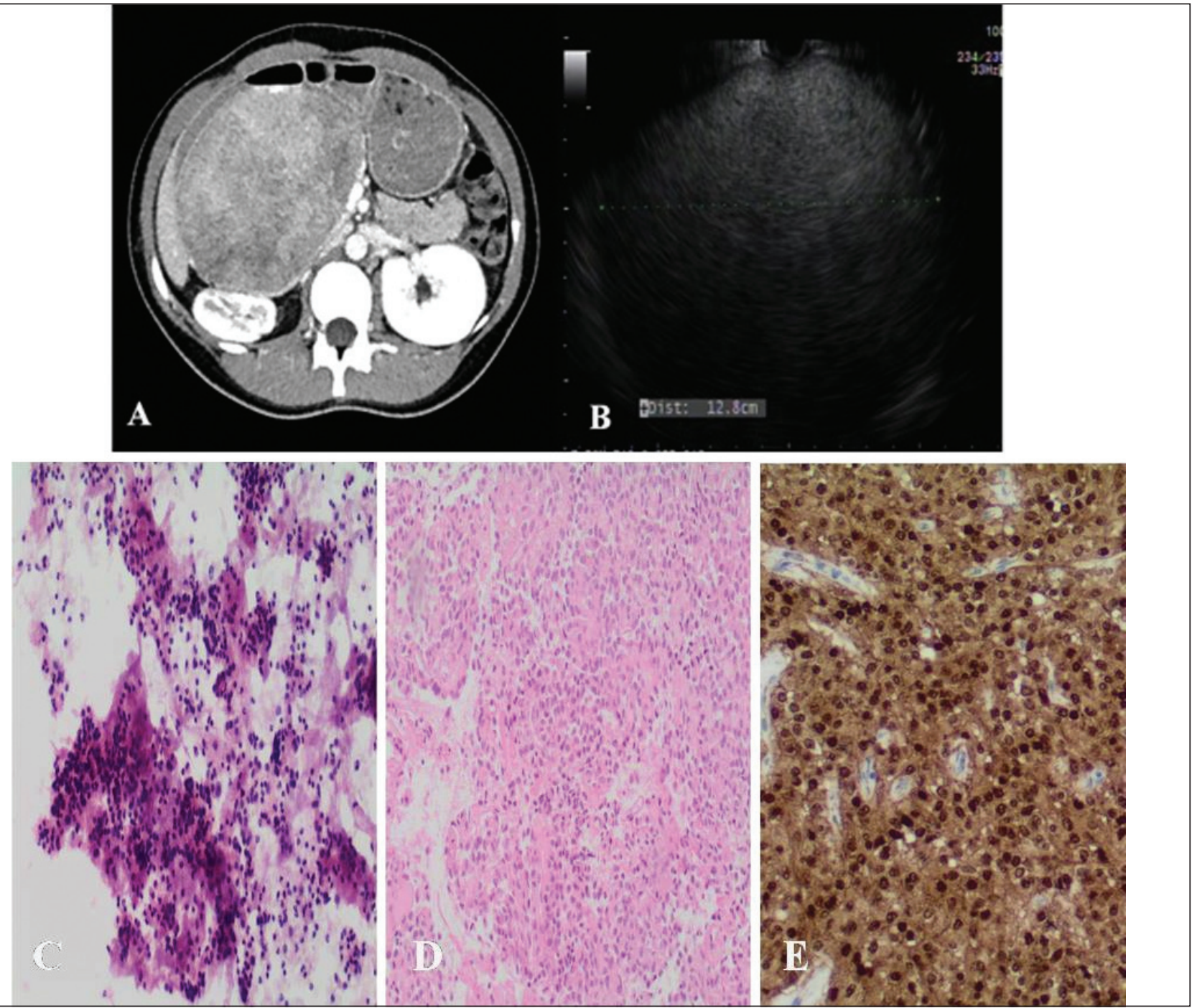

Figure 1) A Abdominal computed tomography scan demonstrating a mass $14 \mathrm{~cm}$ in size in the right upper quadrant. B Endoscopic ultrasound image demonstrating a homogenous solid mass $12.8 \mathrm{~cm}$ in size. C Cellular specimen, composed of neoplastic cells with round to oval nuclei and finely granular chromatin (hematoxylineosin stain, original magnification $\times 200$ ). D Tumour cells surround delicate hyalinized fibrovascular stalks (cell block, hematoxylin-eosin stain, original magnification $\times 200$ ) E The tumour cells show strong nuclear immunoreactivity for beta-catenin (cell block, original magnification $\times 100)$

\footnotetext{
${ }^{1}$ Division of Gastroenterology; ${ }^{2}$ Department of Pathology and Laboratory Medicine, Vancouver General Hospital, University of British Columbia, Vancouver, British Columbia

Correspondence: Dr F Donnellan, Division of Gastroenterology, Vancouver General Hospital, 2775 Laurel Street, Vancouver, British Columbia V5Z 1 M9.

Telephone 778-870-3464, e-mail fdonnellan77@hotmail.com

Received for publication February 5, 2013. Accepted February 19, 2013
} 
SPNs can be located in the tail, head or body of the pancreas. Affected patients are predominantly women of reproductive age who present with nonspecific symptoms: most commonly abdominal pain, nontender epigastric mass, nausea and vomiting. However, a significant proportion $(15 \%)$ of patients are completely asymptomatic, thus making the clinical diagnosis challenging. While SPNs are typically benign lesions, they do have low-grade malignant potential. The recommended treatment is surgical resection, with a five-year survival rate $>95 \%$ (2).

The endoscopic ultrasound appearance of SPNs is nondiagnostic, ranging from well-demarcated, solid-appearing masses, to mixed solid and cystic, and purely cystic lesions. Characteristic cytomorphological features include marked cellularity with pseudopapillary fragments composed of fibrovascular cores lined with discohesive neoplastic cells. Immunohistochemically, the majority are reactive for vimentin, alpha-1 antitrypsin and alpha-1-antichymotrypsin. An alteration in the adenomatous polyposis coli/beta-catenin pathyway has been demonstrated, resulting in nuclear accumulation of beta-catenin in SPNs (3).

\section{REFERENCES}

1. Bosman FT, Carneiro F, Hruban RH, Thiese ND. WHO Classification of Tumors of the Digestive System. Lyon: IARC, 2010.

2. Papavramidis P, Papavramidis S. Solid pseudopapillary tumors of the pancreas: Review of 718 patients reported in English literature. J Am Coll Surg 2005; 200:965-71.

3. Tanaka Y. Frequent beta-catenin mutation and cytoplasmic/nuclear accumulation in pancreatic solid-pseudopapillary neoplasm. Cancer Res 2001;61:8401-4.

The Canadian Journal of Gastroenterology is considering a limited number of submissions for IMAGE OF THE MONTH. These are based on endoscopic, histological, radiological and/or patient images, which must be anonymous with no identifying features visible. The patient must consent to publication and the consent must be submitted with the manuscript. All manuscripts should be practical and relevant to clinical practice, and not simply a case report of an esoteric condition. The text should be brief, structured as CASE PRESENTATION and DISCUSSION, and not more than 700 words in length. A maximum of three images can be submitted and the number of references should not exceed five. The submission may be edited by our editorial team. 


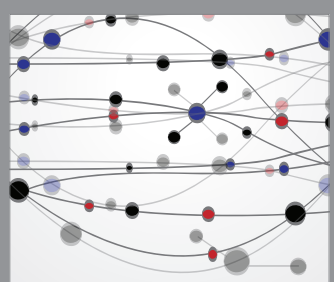

The Scientific World Journal
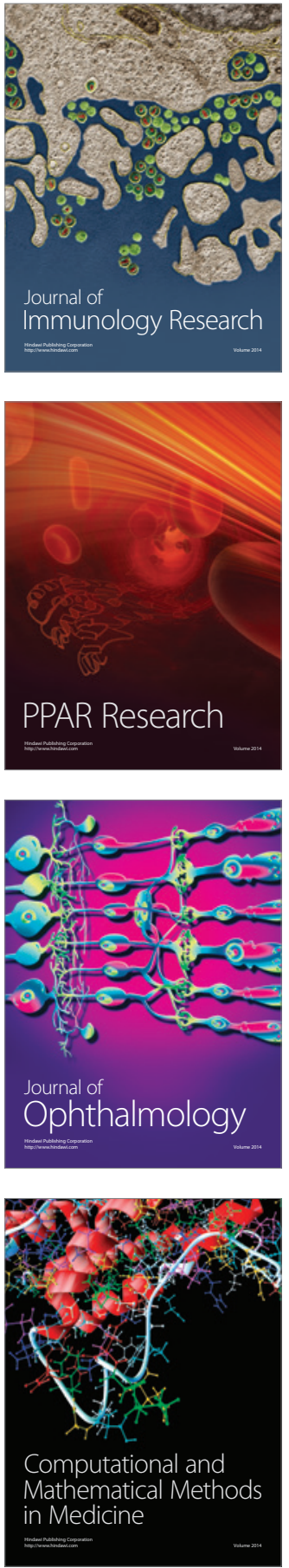

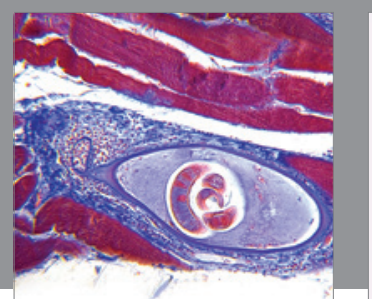

Gastroenterology Research and Practice

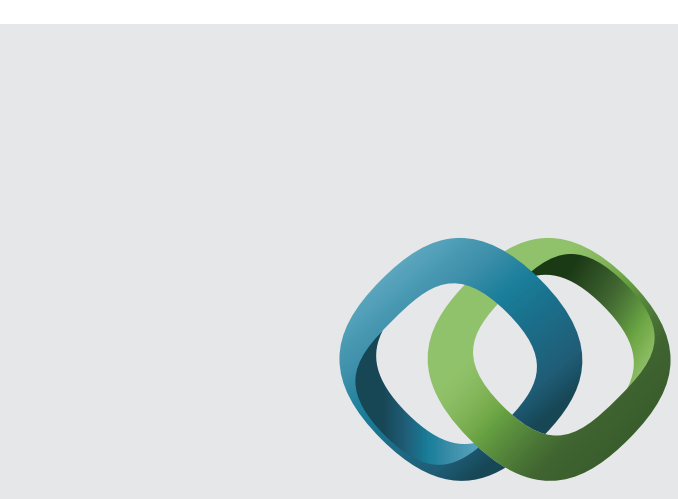

\section{Hindawi}

Submit your manuscripts at

http://www.hindawi.com
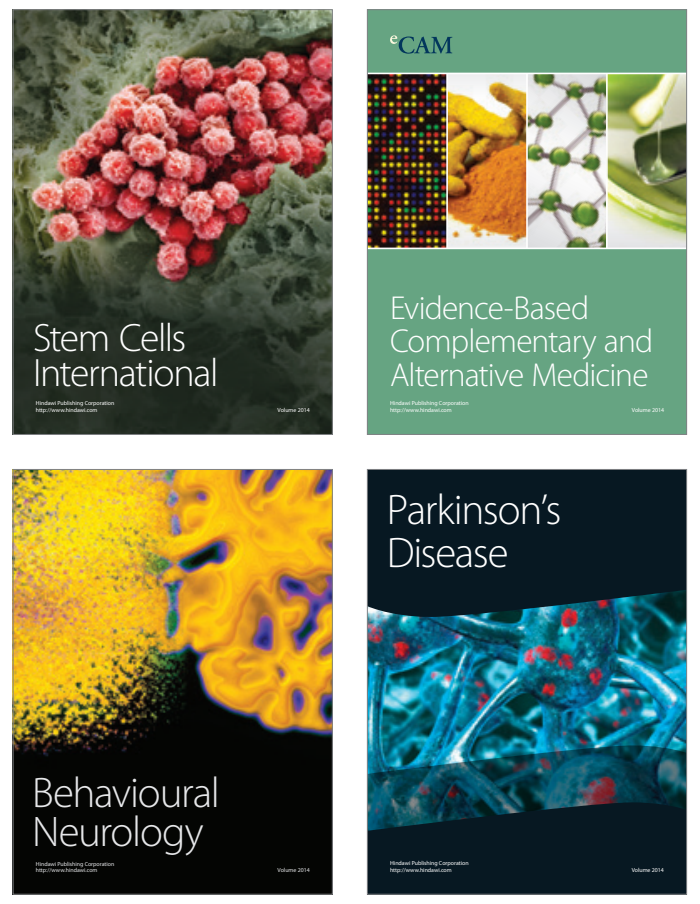
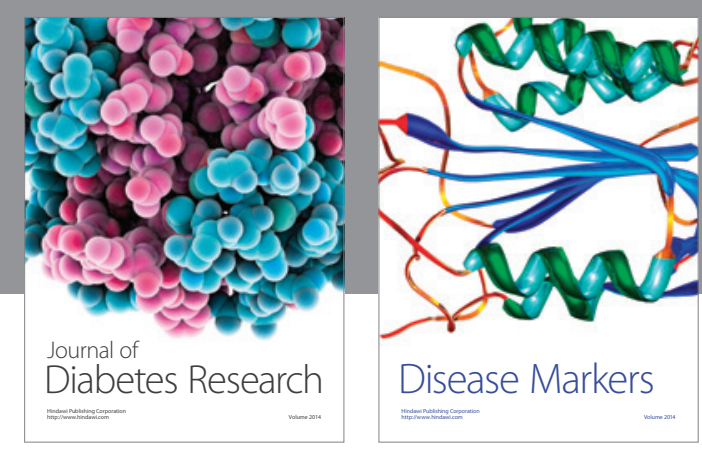

Disease Markers
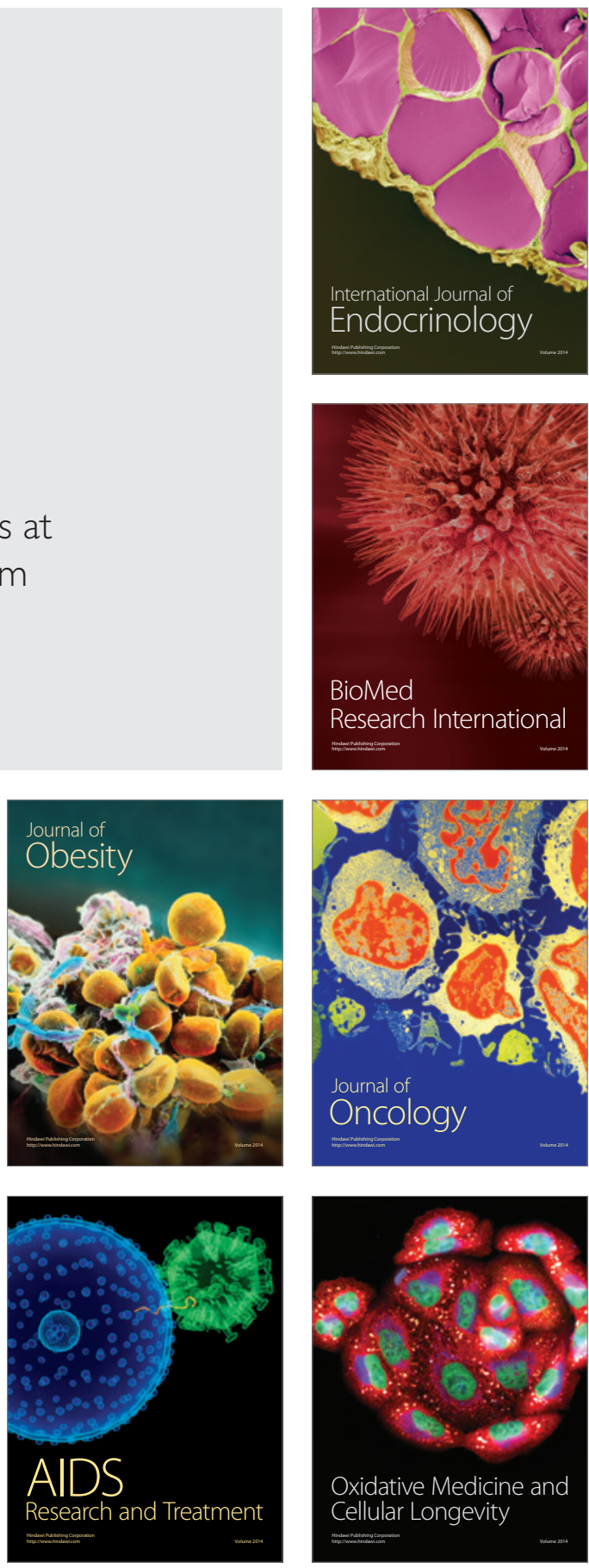